\title{
Mixed Connective Tissue Disease Complicated by Retinal Microvasculopathy and Its Relationship with Fragile-X Syndrome
}

\author{
David Woo $^{1 *}$, Kenneth Ooi ${ }^{2}$, Jennifer Sandbach ${ }^{2}$, Fredrick Joshua ${ }^{2}$ \\ ${ }^{1}$ Department of Ophthalmology, The Canberra Hospital, Canberra, Australia; ${ }^{2}$ Department of Ophthalmology, Prince of Wales Hos- \\ pital, Sydney, Australia. \\ Email: *daviewoo@gmail.com
}

Received April 28 ${ }^{\text {th }}, 2013$; revised May 29 ${ }^{\text {th }}, 2013$; accepted June $15^{\text {th }}, 2013$

Copyright (C) 2013 David Woo et al. This is an open access article distributed under the Creative Commons Attribution License, which permits unrestricted use, distribution, and reproduction in any medium, provided the original work is properly cited.

\begin{abstract}
Purpose: To report an unusual case of retinal microvasculopathy secondary to mixed connective tissue disease (MCTD) on a background history of fragile-X syndrome (FXS). Methods: Case report and literature review. Results: A cotton wool spot was discovered in a 29-year-old female who presented with an ischaemic digit secondary to Raynaud's phenomenon. She also has a background history of MCTD and FXS. Fundus examination and automated perimetry findings were normal. Magnet resonance imaging and computed tomography aortogram did not demonstrate any evidence of vasculitis in the head and neck. She was tested positively for U1-ribonuclear peptide. Interestingly, the re-distribution of Fragile-X related gene 1 has been suggested to trigger autoimmune responses in experiments. This finding makes the case peculiar as it suggests an alternate explanation for this patient's clinical findings. Conclusion: Retinal vasculopathy is a rare complication of MCTD. The background history of FXS potentially highlights an alternate autoimmune pathogenetic mechanism.
\end{abstract}

Keywords: Retinal Microvasculopathy; Mixed Connective Tissue Disease; Fragile-X-Syndrome

\section{Introduction}

Mixed connective tissue disease (MTCD) is a systemic condition immunologically characterised by the presence of $\mathrm{T}$ cells and autoantibodies against U1-ribonuclear peptide (U1RNP). It commonly displays overlapping clinical features of scleroderma (SD), systemic lupus erythematosus (SLE), rheumatoid arthritis and inflammatory myopathy; but the main manifestations include Raynaud's phenomenon, arthralgia, finger or hand oedema, sclerodactyly, myositis and pulmonary involvement [1].

Retinal vasculopathy is rarely reported with MCTD and its pathogenesis remains elusive. It is possible that there may be alternate underlying autoimmune mechanisms at play in the presence of Fragile- $X$ syndrome (FXS). The clinical relation between FXS and MCTD is examined in this article.

\section{Methods}

Case report and literature review.

${ }^{*}$ Corresponding author.

\section{Results}

A 29-year-old anxious female with FXS and MCTD presented with an acutely painful ischaemic left 3rd digit (Figure 1(a)) related to Raynaud's phenomenon, and assorted digit splinter haemorrhages. She was referred to our service for screening prior to hydroxychloroquine commencement; and left thorascopic sympathectomy preoperative workup.

There were no visual complaints at presentation, and her vision was 6/9 bilaterally unaided. No ptosis was evident and her pupils were equal and reactive with no relative afferent pupillary defect. Right fundus examination revealed a single cotton-wool spot (CWS), which raised the concern of retinal microvasculopathy. There were no other significant ocular findings.

Automated perimetry was within normal limits. Photographs were taken (Figure 1(b)) but no fluorescein angiography (FA) was performed due to her anxiety about the risks.

Consequently, magnetic resonant imaging of the brain and arteries were performed, and showed no signs of 


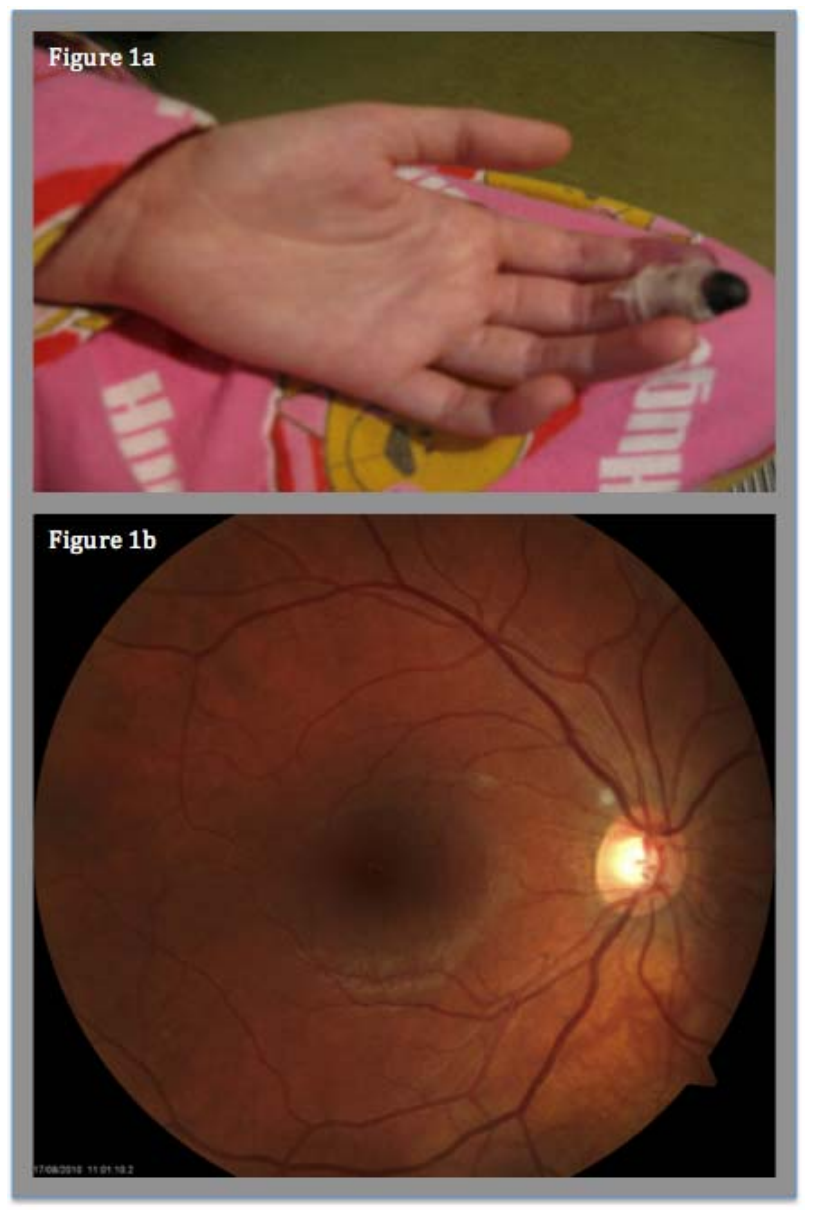

Figure 1. (a) Left ischaemic digit at presentation; (b) Right fundus photograph demonstrating single cotton wool spot.

cerebral vasculitis. No further evidence of stenosis or vasculitis was demonstrated on CT aortogram and duplex ultrasound of the upper limbs and carotids.

Our patient tested positive for U1RNP antibodies, Sjögren's syndrome A antibodies, Smith antibodies and cyclic anti-citrullinated protein. Anti-nuclear antibody titre was $>1: 1520$.

A left thorascopic sympathectomy was performed because the ischaemic digit showed minimal response to epoprostenol treatment. The procedure was uncomplicated and allowed further conservative management without amputation. With still the possibility of a more prevalent systemic small vessel vasculitis she also received three doses of pulsed intravenous methylprednisone $1 \mathrm{gm}$, followed by oral prednisone instead of hydroxychloroquine. She subsequently developed another CWS in the other eye soon thereafter, but to date has not developed extensive pan-retinal vasculitis.

\section{Discussion}

This case is peculiar in two regards, namely, the presence of a retinal microvasculopathy and the associated history of FXS.

Retinal vasculopathy is rarely reported in patients with MCTD. Kraus et al examined the FA findings in patients with MCTD, SLE, and SD. A higher frequency of vascular leakages was observed in the MCTD population (30\%) compared to the other groups [2]. Systemic low dose corticosteroid therapy was found to be beneficial by Mimura [3]; however delays with treatment have lead to severe visual impairment [4].

Our patient's vision was fortunately unaffected despite the threat of overt retinal vasculopathy. Her vision remains stable with ongoing corticosteroid therapy despite development of a further juxtapapillary CWS on the left.

The multifactorial immunopathogenesis of MCTD includes: immune activation via Toll-like receptors, Blymphocytes, CD 4 and 8 T-lymphocytes, autoantibodies, the modification of the RNP antigens and associated RNAs [1]. The last factor is of particular interest in this case. Bolivar et al demonstrated that the Fragile- $X$ related gene 1 (FXR1) in SD is capable of generating an autoimmune response in vitro. In apoptotic cells, FXR1 displaces from its original ribosome-associated cytoplasmic binding site to a more punctate foci, which resemble bleb-like membrane organelles. It was postulated that this re-distribution of FXR1 auto-antigen in apoptosis might form the basis of an autoimmune response with IgG generation to FXR1 [5].

In this case, the cluster of symptoms related to MCTD occurred in an individual with FXS. While this may be a fortuitous appearance of two rare diseases in the same patient, the presence of FXR1 certainly raises the question of an alternate autoimmune mechanism in this patient. Nevertheless from a clinical standpoint it should be emphasized that prompt screening in MCTD may avert sight-threatening vasculitis.

\section{Conclusion}

Retinal vasculopathy is a rare complication of MCTD. The background history of FXS potentially highlights an alternate autoimmune pathogenetic mechanism.

\section{Acknowledgements}

The authors would like to thank the Prince of Wales Ophthalmology clinic for supplying the clinical photographs.

\section{REFERENCES}

[1] R. W. Hoffman and M. E. Maldonada, "Immune Pathogenesis of Mixed Connective Tissue Disease: A Short Analytical Review," Clinical Immunology, Vol. 128, No. 1, 2008, pp. 8-17. doi:10.1016/j.clim.2008.03.461

[2] A. Kraus, G. Cervantes, E. Barojas and D. A. Segovia 
"Retinal Vasculitis in Mixed Connective Tissue Disease. A Fluoroangiographic Study,” Journal of Rheumatology, Vol. 12, No. 6, 1985, pp. 1122-1144.

[3] T. Mimura, T. Usui, S. Amano, S. Yamagami, K. Ono, H. Noma and H. Funatsu, "Retinal Vasculitis and Vitreous Hemorrhage Associated with Mixed Connective Tissue Disease: Retinal Vasculitis in MCTD," International Ophthalmology, Vol. 26, Vol. 4-5, 2005, pp. 159-161.

[4] Y. K. Kim, S. J. Woo, Y. J. Lee and K. H. Park, "Retinal
Vasculopathy Associated with Mixed Connective Tissue Disease,” Ocular Immunology and Inflammation, Vol. 18, No. 1, 2010, pp. 13-15. doi:10.3109/09273940903402629

[5] J. Bolívar, S. Guelman, C. Iglesias, et al., "The Fragile-X-Related Gene FXR1 Is a Human Autoantigen Processed during Apoptosis," The Journal of Biological Chemistry, Vol. 273, No. 127, 1998, pp. 17122-17127. doi:10.1074/jbc.273.27.17122 